\title{
CONTEXTO DE AMBIENTE DE TRABALHO ENTRE ENFERMEIRAS ASSISTENCIAIS EM HOSPITAL UNIVERSITÁRIO
}

\author{
Flávia Boaretto ${ }^{1}$, Maria do Carmo Fernandez Lourenço Haddad², Mariana Angela Rossaneis³ ${ }^{3}$ Raquel Gvozd ${ }^{4}$, \\ Paloma de Souza Cavalcante Pissinati ${ }^{5}$
}

\begin{abstract}
RESUMO: Objetivou-se analisar o ambiente de trabalho favorável à prática profissional de enfermeiros em hospital universitário público. Estudo transversal e quantitativo, realizado com 60 enfermeiros assistenciais, com o questionário Nursing Work Index - Revised. Os dados foram coletados entre novembro 2014 e janeiro de 2015. Dentre os pesquisados, 45 (75\%) enfermeiros consideravam-se satisfeitos com seu trabalho. Todos os 12 setores da instituição apresentaram características favoráveis à prática do enfermeiro nos aspectos de suporte dos gestores, autonomia e controle dos processos de trabalho, sobretudo nas unidades de Transplante de Medula Óssea e a Unidade de Doenças Infectocontagiosas, que se diferenciavam por serem especializadas e com menor de rotatividade de funcionários. A relação médico-enfermeiro foi destacada como um dos pontos críticos, obtendo escores mais insatisfatórios. Conclui-se que a análise de características que favorecem a prática profissional do enfermeiro pode ampliar a discussão de estratégias que contribuam para o trabalho em Enfermagem em serviços de saúde.
\end{abstract}

DESCRITORES: Ambiente de instituições de saúde; Hospitais universitários; Enfermagem; Prática profissional; Ambiente de trabalho.

\section{THE WORK ENVIRONMENT OF NURSES WHO PERFORM CARE ACTIVITIES IN A UNIVERSITY HOSPITAL}

\begin{abstract}
The present study aimed to assess the work environment favorable to the professional practice of nurses in a public university hospital. Cross-sectional quantitative study with 60 care nurses using the Nursing Work Index - Revised questionnaire. The data were collected from November 2014 to January 2015. Also, 45 (75\%) of the nurses who participated in the study satisfied with their work. All the 12 sectors of the institution favored the nursing practice in the following aspects: management support, autonomy and control of work processes, particular in Bone Marrow Transplant units and in the Infectious and Communicable Diseases unit, which, unlike the others, were specialized and had lower employee turnover. According to most respondents, the physician-nurse relationship was poor, unsatisfactory, and obtained low scores. It is concluded that knowledge of the characteristics that favor the exercise of professional nursing activities may broaden the discussion of strategies aimed to improve the nursing work.
\end{abstract}

DESCRIPTORS: Health institutions environment; University hospitals; Nursing; Professional practice; Work environment.

\section{CONTEXTO DE AMBIENTE LABORAL ENTRE ENFERMERAS ASISTENCIALES EN HOSPITAL UNIVERSITARIO}

RESUMEN: Estudio cuyo objetivo fue analizar el ambiente de trabajo favorable a la práctica profesional de enfermeros en hospital universitario público. Trasversal y cuantitativo, el estudio fue realizado con 60 enfermeros asistenciales, por medio de cuestionario Nursing Work Index - Revised. Los datos fueron obtenidos entre noviembre de 2014 y enero de 2015. Entre los investigados, 45 (75\%) enfermeros se dicieron satisfechos con su trabajo. Todos los 12 sectores de la institución presentaron características favorables a la práctica del enfermero en los aspectos de soporte de los gestores, autonomía y control de los procesos de trabajo, sobretodo en las unidades de Trasplante de Médula Osea y la Unidad de Enfermedades Infectocontagiosas, las cuales son distintas por su especialización y su menor rotación de funcionarios. La relación médico-enfermero se destacó como uno de los puntos críticos, con resultados más insatisfactorios. Se concluye que el análisis de características que favorecen la práctica profesional del enfermero puede ampliar la discusión de estrategias que contribuyan para el trabajo en Enfermería en servicios de salud.

DESCRIPTORES: Ambiente de instituciones de salud; Hospitales universitarios; Enfermería; Práctica profesional; Ambiente de trabajo.

${ }^{1}$ Enfermeira. Especialista em Gerência de Serviços de Enfermagem. Londrina, PR, Brasil.

'Enfermeira. Doutora em Enfermagem. Docente de Enfermagem da Universidade Estadual de Londrina. Londrina, PR, Brasil.

${ }^{3}$ Enfermeira. Doutora em Enfermagem. Prefeitura Municipal de Rolândia. Rolândia, PR, Brasil.

${ }^{4}$ Enfermeira. Doutoranda em Enfermagem. Docente de Enfermagem da Universidade Estadual de Londrina. Londrina, PR, Brasil.

${ }^{5}$ Enfermeira. Doutoranda em Enfermagem. Prefeitura Municipal de Rolândia. Rolândia, PR, Brasil.

Autor Correspondente:

Recebido: $19 / 11 / 2015$

Flávia Boaretto

Universidade Estadual de Londrina

R. Elizabeth Kenny, 55 - 86039-460 - Londrina, PR, Brasil

E-mail: flaboaretto@gmail.com 


\section{INTRODUÇÃO}

O ambiente hospitalar constitui um local de trabalho complexo para o exercício profissional. Tal ambiente é reconhecido pelos enfermeiros como lugar que exige elevados níveis de atenção para atender diferentes demandas, pois a prestação de cuidados aos pacientes envolve a complexidade de cada indivíduo ${ }^{(1)}$.

Para se apoderar da prática e prestar assistência de qualidade, o enfermeiro deve estruturar suas ações a partir de processos e valores, tendo domínio sobre o espaço no qual esse cuidado é executado(2). Nesse sentido, o ambiente de trabalho possui grande influência nas atividades coordenadas pelo enfermeiro.

A busca pela melhoria da qualidade da assistência e da segurança do paciente, bem como a implantação de medidas que possam oferecer melhores condições de trabalho para o profissional de saúde, tornaram-se foco dos gestores dos serviços de enfermagem. A maioria das instituições públicas de saúde apresenta em seu contexto a insuficiência de recursos humanos, sobretudo na área de enfermagem, e consequentemente sobrecarga de atividades para os trabalhadores atuantes. Essa situação desencadeou o desenvolvimento de pesquisas nacionais e internacionais direcionadas a reflexão sobre o ambiente de trabalho do enfermeiro, diante de sua influência sobre as atividades profissionais $^{(3)}$.

O ambiente de trabalho favorável ao enfermeiro oferece subsídios para o desenvolvimento do exercício profissional e apresenta três características fundamentais: a autonomia, o controle sobre o ambiente e a boa relação entre médicos e enfermeiros ${ }^{(4)}$. Portanto, para fundamentar sua prática como gestor de serviços, faz-se necessário promover a avaliação da qualidade desses pilares que sustentam a atuação das equipes ${ }^{(5)}$.

Estudo evidenciou que determinados fatores do ambiente podem influenciar os cuidados de enfermagem, pois existe uma relação entre o local de trabalho, as ações realizadas e as possíveis consequências decorrentes da assistência prestada ao paciente ${ }^{(6)}$.

Destaca-se que quando, no ambiente profissional do enfermeiro, surgem características como a valorização profissional, boas relações interpessoais, gestão de qualidade e adequada remuneração, pode haver uma diminuição do nível de Burnout. Esta se constitui em um transtorno ocupacional desenvolvido em função de sobrecargas e más condições de trabalho que, em conjunto com outros fatores, contribuem para a despersonalização, baixa realização profissional e exaustão emocional dos trabalhadores ${ }^{(5,7)}$.

Quando a instituição proporciona um ambiente de trabalho favorável, apresentará menores taxas de absenteísmos e rotatividade entre os trabalhadores, além de contribuir para a diminuição das taxas de mortalidade e elevação do índice de satisfação dos pacientes com a assistência recebida ${ }^{(5,7)}$.

Ressalta-se que o enfermeiro tem papel fundamental para propiciar assistência com maior qualidade aos pacientes. Dessa forma, promover a autonomia desses profissionais e o domínio sobre o seu local de trabalho contribuem para um ambiente favorável ao exercício profissional. Além disso, a relação com a equipe médica, bem como com outros profissionais envolvidos na assistência, deve ser fundamentada no respeito aos conhecimentos e habilidades requeridas de cada profissional e no favorecimento do processo de comunicação para efetividade do cuidado ${ }^{(4)}$.

Para tanto se pode utilizar alguns instrumentos, dentre os quais se destaca a versão brasileira adaptada e validada do Nursing Work Index - Revised (NWI-R), que mensura a presença de características favoráveis à prática profissional do enfermeiro no seu ambiente de trabalho ${ }^{(4)}$. O NWI-R é uma medida amplamente utilizada para avaliar se os ambientes estão adequados à prática profissional do enfermeiro, sendo recomenda por várias organizações dos Estados Unidos que promovem cuidados de saúde de qualidade ${ }^{(8)}$.

Ao analisar o ambiente, é possível desenvolver alternativas que promovam maior satisfação dos profissionais e, assim, proporcionar benefícios aos enfermeiros, pacientes e instituição. Realizar melhorias no local de trabalho contribui para a segurança do paciente e dos profissionais envolvidos 
nos cuidados, e dentre os instrumentos utilizados para tal avaliação, o NWI-R apresenta maior utilidade devido ao seu conjunto de critérios.

Portanto, este estudo objetivou analisar o ambiente de trabalho para prática assistencial do enfermeiro em um hospital universitário público.

\section{MÉTODO}

Trata-se de uma pesquisa transversal, descritiva, de abordagem quantitativa, realizada em um hospital universitário público localizado no norte do estado do Paraná, centro de referência regional para o Sistema Único de Saúde (SUS).

Os dados foram coletados entre os meses de novembro de 2014 e janeiro de 2015. Neste período, a instituição de estudo possuía 117 enfermeiros, sendo incluídos aqueles que atuavam diretamente na assistência ao paciente nas unidades de pronto socorro, médico-cirúrgica masculina e feminina, doenças infectocontagiosas, maternidade, unidade de terapia adulto, infantil e neonatal, centro cirúrgico, centro de tratamento de queimados, pediatria e transplante de medula óssea com mais de um ano de trabalho na instituição. Foram excluídos do estudo os enfermeiros que estavam em férias ou licenças especiais, e que não atuavam diretamente na assistência, resultando em uma população de 73 profissionais, dos quais 60 aceitaram participar do estudo e responderam ao questionário aplicado, obtendo $82,19 \%$ de participação.

Os enfermeiros que aceitaram participar do estudo, mediante assinatura do termo de consentimento livre e esclarecido, responderam a dois instrumentos, sendo o primeiro um questionário sociodemográfico e ocupacional, composto por variáveis referentes ao sexo, faixa etária, estado civil, tempo de experiência profissional e de trabalho na instituição, nível de formação profissional, forma de admissão na instituição, número de pacientes sob a responsabilidade do enfermeiro, área de atuação e turno de trabalho.

O segundo instrumento, denominado "Nursing Work Index - Revised" (NWI-R) é um questionário com 57 itens, que mensura a presença de características que favorecem a prática profissional do enfermeiro. Para esse feito, dos 57 itens, 15 foram distribuídos de forma conceitual em três subescalas: autonomia, controle e relação entre médicos e enfermeiros, dentre esses 15 itens, dez foram agrupados para derivar a quarta subescala, que avalia o suporte organizacional $(2,4,9)$.

As subescalas "autonomia", formada por cinco itens, e "controle", com sete itens, representam a liberdade que o enfermeiro possui para solucionar problemas; a subescala "relações entre médicos e enfermeiros", possui três itens e envolve comunicação efetiva em saúde, por meio da qual deve ocorrer o respeito mútuo na busca pelo alcance de um único objetivo, o cuidado prestado ao paciente; e o "suporte organizacional", composto por dez itens derivados das subescalas anteriores, está relacionado às situações em que o enfermeiro recebe o suporte da instituição para desenvolver sua prática profissional ${ }^{(9)}$.

A escala utiliza a medida do tipo Likert que varia de um a quatro pontos. O participante é convidado a responder se concorda ou não com a afirmativa "esse fator está presente no meu trabalho diário", com as seguintes alternativas: concordo totalmente (um ponto); concordo parcialmente (dois pontos); discordo parcialmente (três pontos); e discordo totalmente (quatro pontos). Quanto menor a pontuação, maior a presença de atributos favoráveis à prática do enfermeiro ${ }^{(2,9)}$.

A análise estatística dos dados foi realizada em um programa informatizado para este fim e foram apresentadas em estatística descritiva com obtenção de média, desvio-padrão, frequências relativas e absolutas.

O projeto foi aprovado em outubro de 2014 pelo Comitê de Ética em Pesquisa Envolvendo Seres Humanos, conforme parecer $n^{\circ} 821.558$. 
Dos 60 entrevistados, 47 (78,3\%) eram do sexo feminino, possuíam, em média, 45,2 anos e mediana de 43 anos. Em relação ao estado civil, 34 (56,7\%) participantes possuíam um relacionamento estável, $13(21,7 \%)$ eram solteiros, oito (13,3\%) divorciados, três $(5 \%)$ separados, um $(1,7 \%)$ viúvo e um $(1,7 \%)$ referiu não se enquadrar em nenhum destes relacionamentos.

Identificou-se que os participantes possuíam, em média, 18,9 anos de experiência como enfermeiros, com mínimo de um ano e seis meses e máximo de 34 anos. Já o tempo médio de trabalho na instituição foi de 14 anos, com mediana de 12,9 anos e variação de um a 43,1 anos.

Quanto à formação profissional, 41 (68,3\%) entrevistados possuíam especialização na área, 15 (25\%) eram mestres e dois $(3,3 \%)$ doutores. Apenas um enfermeiro $(1,7 \%)$ referiu possuir a graduação e um $(1,7 \%)$ não respondeu esta questão.

No que se refere à forma de admissão, 41 enfermeiros (68,3\%) foram admitidos por concurso público e $19(31,7 \%)$ ingressaram por meio de processo seletivo simplificado, ou seja, possuíam um vínculo trabalhista por tempo determinado, permanecendo no máximo por dois anos na instituição. A maioria, 44 enfermeiros (73,3\%), informou não possuir outro vínculo empregatício e, dentre os 16 (26,7\%) que possuíam outro trabalho, dez (62,5\%) eram contratados por processo seletivo.

Em relação ao número de pacientes sob a responsabilidade do enfermeiro durante o seu turno de trabalho, cada profissional responsabilizava-se, em média, por 23 pacientes, com mediana de 17 pacientes.

Quanto à área de atuação, 12 (20\%) participantes atuavam na unidade de terapia intensiva adulto, dez $(16,7 \%)$ no pronto socorro, sete $(11,7 \%)$ no centro de tratamento de queimados, cinco $(8,3 \%)$ no centro cirúrgico, quatro $(6,7 \%)$ na unidade médico-cirúrgica feminina, quatro $(6,7 \%)$ na unidade médicocirúrgica masculina, quatro $(6,7 \%)$ na maternidade, três (5\%) na unidade de doenças infectocontagiosas, três $(5 \%)$ na unidade de terapia intensiva neonatal, três (5\%) na pediatria, três $(5 \%)$ na unidade de transplante de medula óssea e dois $(3,3 \%)$ na unidade de terapia intensiva pediátrica.

Ainda, em todos os setores pesquisados o percentual de participantes foi representativo da população de enfermeiros, obtendo amostras superiores a $60 \%$, conforme Tabela 1.

Tabela 1 - Representatividade da amostra de enfermeiros de um hospital universitário público, de acordo com o setor de trabalho. Londrina, PR, Brasil, 2015

\begin{tabular}{lccc} 
Setor & $\begin{array}{c}\text { População de } \\
\text { enfermeiros }\end{array}$ & \multicolumn{2}{c}{$\begin{array}{c}\text { Amostra de enfermeiros } \\
\text { participantes do estudo }\end{array}$} \\
\cline { 2 - 4 } & & $\mathbf{n}$ & \% \\
\hline Pronto Socorro & 12 & 10 & 83,3 \\
\hline Unidade Feminina & 6 & 4 & 66,7 \\
\hline Unidade Masculina & 5 & 4 & 80 \\
\hline Doenças Infectocontagiosas & 4 & 3 & 75 \\
\hline Maternidade & 5 & 4 & 80 \\
\hline Unidade de Terapia Intensiva Adulto (UTI-Adulto) & 12 & 12 & 100 \\
\hline Centro Cirúrgico & 6 & 5 & 83,3 \\
\hline Centro Trat. Queimados & 8 & 7 & 87,5 \\
\hline Unidade Pediátrica & 5 & 3 & 60 \\
\hline Unidade de Terapia Intensiva Neonatal (UTI-Neonatal) & 4 & 3 & 75 \\
\hline Unidade de Terapia Intensiva Pediátrica (UTI-Pediátrica) & 3 & 2 & 66,7 \\
\hline Transplante de Medula Óssea & 3 & 3 & 100 \\
\hline Total & $\mathbf{7 3}$ & $\mathbf{6 0}$ & $\mathbf{8 2 , 2}$
\end{tabular}


No que se refere ao turno de trabalho, $22(36,7 \%)$ participantes atuavam no período noturno, 17 $(28,3 \%)$ trabalhavam no período da manhã, $14(23,3 \%)$ à tarde e sete $(11,7 \%)$ informaram atuar em outros turnos.

Destaca-se que 45 (75\%) enfermeiros consideravam-se satisfeitos com seu trabalho, nove (15\%) muito satisfeitos, três (5\%) insatisfeitos e três (5\%) muito insatisfeitos. Já a qualidade do trabalho prestado foi avaliada por 14 (23,3\%) participantes como muito boa, 14 (23,3\%) como boa, um indivíduo $(1,7 \%)$ a considerou ruim e nenhum a classificou como muito ruim.

As características do ambiente de trabalho favoráveis à prática profissional foram categorizadas em quatro subescalas, com destaque para a autonomia, com uma média geral de 2,02 pontos; seguida pelo suporte organizacional com 2,17 pontos; relação médico e enfermeiros, 2,23 pontos; e o controle sobre o ambiente de trabalho com média de 2,29 pontos, conforme Tabelas 2 e 3.

Observa-se que as unidades mais favoráveis à autonomia do enfermeiro foram as de transplante de medula óssea, doenças infectocontagiosas, unidade pediátrica, unidade de terapia intensiva - adulto e centro de tratamento de queimados.

Quanto à relação entre médicos e enfermeiros, os setores que apresentaram melhores escores

Tabela 2 - Características do ambiente de trabalho favoráveis à prática profissional de enfermeiros de um hospital universitário público em relação à autonomia e relação médico/enfermeiro, de acordo com o setor de trabalho. Londrina, PR, Brasil, 2015

\begin{tabular}{|c|c|c|c|c|c|}
\hline Escore & Setor & Mínimo & Máximo & Média & $\begin{array}{l}\text { Desvio } \\
\text { Padrão }\end{array}$ \\
\hline \multirow[t]{13}{*}{ NWI-R Autonomia } & Geral & 1 & 3,8 & 2,02 & 0,6 \\
\hline & Pronto Socorro & 1,4 & 2,8 & 2,04 & 0,46 \\
\hline & Unidade Feminina & 2 & 3,2 & 2,45 & 0,57 \\
\hline & Unidade Masculina & 1,4 & 3,4 & 2,45 & 0,82 \\
\hline & Doenças Infectocontagiosas & 1,2 & 2,2 & 1,53 & 0,57 \\
\hline & Maternidade & 1,4 & 3 & 2,15 & 0,66 \\
\hline & UTI-Adulto & 1 & 3,8 & 1,91 & 0,74 \\
\hline & Centro Cirúrgico & 1,6 & 3 & 2,28 & 0,6 \\
\hline & Centro Trat. Queimados & 1,4 & 2,4 & 1,91 & 0,32 \\
\hline & Unidade Pediátrica & 1,6 & 2,2 & 1,8 & 0,34 \\
\hline & UTI-Neonatal & 1,8 & 2,6 & 2,26 & 0,41 \\
\hline & UTI-Pediátrica & 2,2 & 2,6 & 2,4 & 0,28 \\
\hline & Transplante Medula Óssea & 1 & 1,4 & 1,2 & 0,2 \\
\hline \multirow{13}{*}{$\begin{array}{l}\text { NWI-R Relação Médico e } \\
\text { Enfermeiros }\end{array}$} & Geral & 1 & 3,67 & 2,23 & 0,69 \\
\hline & Pronto Socorro & 1,67 & 3,33 & 2,26 & 0,51 \\
\hline & Unidade Feminina & 2 & 3,67 & 2,66 & 0,81 \\
\hline & Unidade Masculina & 2,67 & 3,67 & 3,08 & 0,5 \\
\hline & Doenças Infectocontagiosas & 1 & 2,33 & 1,77 & 0,69 \\
\hline & Maternidade & 1,67 & 3,67 & 2,58 & 0,91 \\
\hline & UTI-Adulto & 1 & 3,67 & 1,88 & 0,72 \\
\hline & Centro Cirúrgico & 2 & 3 & 2,46 & 0,44 \\
\hline & Centro Trat. Queimados & 1,33 & 2,67 & 2,23 & 0,49 \\
\hline & Unidade Pediátrica & 2 & 2,67 & 2,33 & 0,33 \\
\hline & UTI-Neonatal & 1,67 & 2,33 & 2 & 0,33 \\
\hline & UTI-Pediátrica & 2,67 & 3 & 2,83 & 0,23 \\
\hline & Transplante Medula Óssea & 1 & 1,33 & 1,11 & 0,19 \\
\hline
\end{tabular}


foram a unidades infectocontagiosas (1,77 pontos) e de terapia intensiva - adulto (1,88 pontos), já o escore menos favorável ocorreu na unidade médico-cirúrgica masculina (3,08 pontos).

No que diz respeito ao controle sobre o ambiente de trabalho, os setores com melhor avaliação pelos enfermeiros foram às unidades de transplante de medula óssea (1,14 pontos), doenças infectocontagiosas (1,90 pontos) e o centro de tratamento de queimados (1,91 pontos). Já na última subescala denominada "suporte organizacional", que envolve o apoio fornecido pela instituição aos enfermeiros na prestação dos cuidados, os setores de transplante de medula óssea (1,10 pontos), doenças infectocontagiosas (1,76 pontos) e centro de tratamento de queimados (1,92 pontos) apresentaram novamente os melhores índices de acordo com a avaliação dos enfermeiros.

Os resultados das subescalas de acordo com a avaliação por sexo demonstraram que os homens consideraram o ambiente de trabalho mais favorável à prática profissional do que as mulheres, com destaque para a autonomia, que obteve melhor média para o sexo masculino, 1,86 pontos, como exposto na Tabela 4.

Os homens também apresentaram maior satisfação profissional quando comparados ao sexo feminino, sendo que $12(92,3 \%)$ sentiam-se satisfeitos ou muito satisfeitos, assim como as mulheres no percentual de $42(89,4 \%)$.

Tabela 3 - Características do ambiente de trabalho favoráveis à prática profissional de enfermeiros de um hospital universitário público em relação ao controle sobre o ambiente e suporte organizacional, de acordo com o setor de trabalho. Londrina, PR, Brasil, 2015

\begin{tabular}{|c|c|c|c|c|c|}
\hline Escore & Setor & Mínimo & Máximo & Média & $\begin{array}{l}\text { Desvio } \\
\text { Padrão } \\
\end{array}$ \\
\hline \multirow{13}{*}{$\begin{array}{l}\text { NWI-R } \\
\text { Controle sobre o ambiente }\end{array}$} & Geral & 1 & 3,57 & 2,29 & 0,63 \\
\hline & Pronto Socorro & 1,71 & 3,14 & 2,44 & 0,43 \\
\hline & Unidade Feminina & 1,71 & 3,43 & 2,57 & 0,73 \\
\hline & Unidade Masculina & 2,14 & 3,57 & 2,67 & 0,62 \\
\hline & Doenças Infectocontagiosas & 1,57 & 2,14 & 1,9 & 0,29 \\
\hline & Maternidade & 1,71 & 3,43 & 2,57 & 0,84 \\
\hline & UTI-Adulto & 1,14 & 3,14 & 2,05 & 0,58 \\
\hline & Centro Cirúrgico & 1,57 & 3,29 & 2,71 & 0,66 \\
\hline & Centro Trat. Queimados & 1,43 & 2,14 & 1,91 & 0,25 \\
\hline & Unidade Pediátrica & 2,29 & 2,71 & 2,5 & 0,3 \\
\hline & UTI-Neonatal & 2,14 & 3,29 & 2,66 & 0,57 \\
\hline & UTI-Pediátrica & 2,71 & 3 & 2,85 & 0,2 \\
\hline & Transplante Medula Óssea & 1 & 1,29 & 1,14 & 0,14 \\
\hline \multirow{13}{*}{$\begin{array}{l}\text { NWI-R } \\
\text { Suporte Organizacional }\end{array}$} & Geral & 1 & 3,4 & 2,17 & 0,54 \\
\hline & Pronto Socorro & 1,8 & 2,9 & 2,25 & 0,51 \\
\hline & Unidade Feminina & 1,8 & 3,3 & 2,47 & 0,66 \\
\hline & Unidade Masculina & 2,1 & 3,4 & 2,6 & 0,55 \\
\hline & Doenças Infectocontagiosas & 1,3 & 2,2 & 1,76 & 0,45 \\
\hline & Maternidade & 1,8 & 3,1 & 2,42 & 0,53 \\
\hline & UTI-Adulto & 1,1 & 3,4 & 2,01 & 0,63 \\
\hline & Centro Cirúrgico & 1,9 & 3 & 2,56 & 0,42 \\
\hline & Centro Trat. Queimados & 1,5 & 2,2 & 1,92 & 0,22 \\
\hline & Unidade Pediátrica & 2 & 2,4 & 2,13 & 0,23 \\
\hline & UTI-Neonatal & 2,3 & 2,5 & 2,43 & 0,11 \\
\hline & UTI-Pediátrica & 2,6 & 2,7 & 2,65 & 0,7 \\
\hline & Transplante Medula Óssea & 1 & 1,2 & 1,1 & 0,1 \\
\hline
\end{tabular}


Tabela 4 - Características do ambiente de trabalho favoráveis à prática profissional de enfermeiros de um hospital universitário público, de acordo com o sexo dos participantes. Londrina, PR, Brasil, 2015

\begin{tabular}{llcccc} 
Escore & Sexo & Mínimo & Máximo & Média & $\begin{array}{c}\text { Desvio } \\
\text { Padrão }\end{array}$ \\
\hline Autonomia & Feminino & 1 & 3,8 & 2,07 & 0,62 \\
\cline { 2 - 6 } & Masculino & 1 & 2,6 & 1,86 & 0,5 \\
\hline Controle sobre o ambiente & Feminino & 1 & 3,57 & 2,33 & 0,65 \\
\cline { 2 - 6 } & Masculino & 1,29 & 3,14 & 2,13 & 0,53 \\
\hline Relação Médico e Enfermeiros & Feminino & 1 & 3,67 & 2,28 & 0,72 \\
\cline { 2 - 6 } & Masculino & 1 & 3,33 & 2,05 & 0,57 \\
\hline Suporte Organizacional & Feminino & 1 & 3,4 & 2,22 & 0,57 \\
\cline { 2 - 6 } & Masculino & 1,1 & 2,6 & 2 & 0,42
\end{tabular}

\section{- DISCUSSÃO}

O predomínio do sexo feminino na população estudada se assemelha aos resultados da pesquisa do Perfil da enfermagem no Brasil, realizado pelo Conselho Federal de Enfermagem (COFEN) e Fundação Oswaldo Cruz (Fiocruz) em 2010 e divulgada em maio de 2015, no qual havia um predomínio de 87,24\% de mulheres, de um total de 1.449 .583 profissionais, o que corresponde a $64,7 \%$ da força de trabalho na saúde ${ }^{(10)}$.

A maioria dos participantes $(57,6 \%)$ estava em um relacionamento estável, fato que pode ser positivo para esses trabalhadores, visto que o apoio emocional decorrente da relação estabelecida com o cônjuge contribui para o enfrentamento dos problemas profissionais, além de estar associado a menor ocorrência de transtornos psicológicos, como a síndrome de Burnout em enfermeiros ${ }^{(11)}$.

Os resultados revelaram que muitos trabalhadores buscaram a pós-graduação em enfermagem após ingressarem na instituição, dado que pode estar associado ao interesse em progredir profissionalmente e alcançar melhores condições financeiras, mesmo diante do desafio de conciliar estudos e trabalho ${ }^{(12)}$. Além disso, a especialização lato ou stricto sensu podem ampliar os conhecimentos que contribuem para o desenvolvimento de competências assistenciais e gerenciais do enfermeiro que, assim, tende a reagir de maneira mais resolutiva às dificuldades encontradas no ambiente de trabalho.

No presente estudo, a maior parte da amostra referiu estar satisfeita ou muito satisfeita com seu trabalho, resultado que demonstra que os enfermeiros apresentavam uma visão positiva de suas competências no seu ambiente de trabalho, tornando-o favorável à sua prática. De forma semelhante, pesquisa desenvolvida com enfermeiros revelou que eles se sentiam mais satisfeitos com os aspectos inerentes ao seu trabalho, como o reconhecimento e a responsabilidade, do que aqueles que envolvem salário, qualidade de supervisão, relação com a equipe de trabalho e as próprias condições de trabalho ${ }^{(13)}$. O profissional satisfeito tende a ter uma boa relação com a instituição, empenhando maior qualidade em suas ações, sendo que os maiores beneficiados serão os pacientes.

A elevada satisfação profissional expressada por $15 \%$ dos participantes também pode estar relacionada à presença de características favoráveis vinculadas ao suporte organizacional na instituição em estudo. Estudo, realizado na Espanha, em 2013, com 111 enfermeiros, revelou que a presença do apoio ou suporte da organização contribuía para que o profissional adquirisse uma visão positiva do ambiente laboral, além de ressaltar a importância do suporte dos gestores para o desenvolvimento de um local de trabalho favorável à prática do enfermeiro ${ }^{(14)}$, pois ao reconhecer as competências do profissional e fornecer maior apoio, contribuem para a autonomia no processo de tomada de decisão e favorecem a sua responsabilização pelo trabalho e satisfação com os resultados alcançados.

Em relação à autonomia profissional, a maioria dos setores em estudo não apresentou características favoráveis, sobretudo as unidades masculina, feminina e UTI pediátrica. Ressalta-se que o exercício da autonomia contribui para a solidificação do perfil profissional do enfermeiro, contudo, trata-se de uma 
tarefa árdua, pois requer o desenvolvimento da capacidade de tomada de decisão, de se relacionar com o próximo e de respeitar a atuação dos demais membros da equipe multiprofissional (14-15), fatores de difícil implementação nos setores mencionados, devido à alta complexidade da assistência prestada e à elevada demanda de pacientes atendidos diariamente.

Os resultados desfavoráveis referentes à relação entre médicos e enfermeiros, evidenciado em nove dos 12 setores pesquisados, dentre os quais se destaca a unidade masculina, reforçam a premissa de que estes atuam de forma interdependente nas organizações hospitalares, e mantém estreito vínculo, portanto, tendem a desencadear conflitos entre as profissões. As relações conflituosas são um obstáculo à melhoria da qualidade da assistência prestada ${ }^{(16)}$. Além disso, a interação entre esses profissionais deve ser adequada para a tomada de decisão no cuidado ao paciente. O bom relacionamento auxilia na melhoria da qualidade da assistência prestada aos clientes, influencia o clima da prática do enfermeiro, que sofre interferência tanto do espaço físico quanto do ambiente interno de trabalho ${ }^{(13)}$.

Quanto ao controle sobre o ambiente de trabalho, os setores com melhor avaliação pelos enfermeiros foram as unidades de transplante de medula óssea, doenças infectocontagiosas e o centro de tratamento de queimados. Estes resultados podem estar relacionados ao fato dessas unidades serem menores, com equipe multiprofissional estruturada que atende a uma população específica de pacientes, além de possuírem uma rotatividade menor de funcionários. Ao propiciar que os enfermeiros possuam controle sobre o ambiente de trabalho, bem como suporte organizacional, a instituição permite que o profissional exerça seu papel e administre a unidade com critérios bem definidos ${ }^{(17)}$.

Ressalta-se que um ambiente favorável à prática de enfermeiros pode ser modificado e aprimorado por meio de ações administrativas. Estudo realizado em 2006, em Nova Jersey, nos Estados Unidos da América, com 863 enfermeiros, demonstrou que um local de trabalho adequado à atuação da enfermagem aumentava o nível da qualidade da assistência e contribuía para a redução das taxas de mortalidade e de eventos adversos aos pacientes ${ }^{(18)}$.

No presente estudo, os homens consideraram o ambiente de trabalho mais favorável à prática profissional e sentiam-se satisfeitos, sobretudo no que se referia ao exercício da autonomia. Esse resultado aponta a necessidade dos gestores institucionais direcionarem as ações de melhoria do ambiente laboral(18) de acordo com as características sociodemográficas e ocupacionais dos trabalhadores, uma vez que estas podem influenciar a percepção sobre os aspectos positivos e negativos existentes em seu local de trabalho.

Ao se preocuparem com as características favoráveis ao ambiente de trabalho do enfermeiro, as instituições de saúde poderão oferecer um melhor local de trabalho aos seus funcionários. Essa estratégia contribuirá para a redução dos gastos com absenteísmos e rotatividade, menores níveis de exaustão emocional e maior satisfação dos profissionais para se produzir ambientes favoráveis. A instituição deve requerer medidas válidas e confiáveis, para que o processo seja efetivo.

Salienta-se que quanto mais o trabalhador estiver insatisfeito em seu ambiente de trabalho, maiores serão os índices de demissões. Nesse sentido, o fornecimento de suporte organizacional torna-se uma importante estratégia gerencial para que os profissionais sintam-se valorizados e reconhecidos, fatores que podem contribuir para o aumento da satisfação e para a retenção dos enfermeiros nas instituições.

\section{CONCLUSÃO}

Todas as unidades da instituição possuíam características favoráveis à prática profissional. O suporte dos gestores, a autonomia e o controle dos processos de trabalho foram avaliados pelos enfermeiros com algumas características favoráveis para um ambiente adequado à sua prática assistencial. Contudo, a relação médico-enfermeiro ainda é um desafio a ser superado para a melhoria da comunicação entre a equipe multidisciplinar e a garantia da segurança do paciente. Também foi possível verificar que homens possuíam uma melhor média nas características favoráveis à prática profissional em relação às mulheres, bem como uma maior satisfação profissional.

Os resultados fornecem subsídios para locais semelhantes ao investigado e contribui para a 
avaliação do ambiente, adequação da estrutura e do processo de trabalho nos diferentes setores, além de ampliar a discussão sobre os fatores considerados favoráveis à prática profissional. Com isso, as instituições poderão desenvolver estratégias que contribuam com boas práticas no trabalho do enfermeiro, propiciando uma melhora na qualidade da assistência prestada aos pacientes.

\section{REFERÊNCIAS}

1. Santos LSC, Guirardello EB. Nurses attention demands in work setting. Rev Latino-Am Enfermagem. [Internet] 2007;15(1) [acesso em 23 dez 2015]. Disponível: http://dx.doi.org/10.1590/S0104-11692007000100005.

2. Gasparino RC, Guirardello EB. Translation and cross-cultural adaptation of the "Nursing Work Index - Revised" into Brazilian Portuguese. Acta paul. enferm. [Internet] 2009;22(3) [acesso em 23 dez 2015]. Disponível: http:// dx.doi.org/10.1590/S0103-21002009000300007.

3. Roscani ANCP, Guirardello EB. Attention Demands in the Workplace and the Capacity to Direct Attention of Nurses. Rev. Latino-Am Enfermagem. [Internet] 2010;18(4) [acesso em 23 dez 2015]. Disponível: http://dx.doi. org/10.1590/S0104-11692010000400017.

4. Gasparino RC. Adaptação cultural e validação do instrumento Nursing Work Index - Revised para a cultura brasileira [dissertação]. Campinas (SP): Universidade Estadual de Campinas; 2008.

5. Balsanelli AP, Cunha ICKO. The work environment in public and private intensive care units. Acta paul. enferm. [Internet] 2013;26(6) [acesso em 23 dez 2015]. Disponível: http://dx.doi.org/10.1590/S0103-21002013000600009.

6. Ferreira MRSCF, Martins JJPA. Study of adaptation and validation of the Practice environment scale of the Nursing work index for the portuguese reality. Rev Esc Enferm USP. [Internet] 2014;48(4) [acesso em 23 dez 2015]. Disponível: http://dx.doi.org/10.1590/S0080-623420140000400017.

7. Portela NLC, Pedrosa AO, Cunha JDS, Monte LRS, Gomes RNS, Lago EC. Síndrome de Burnout em profissionais de enfermagem de serviços de urgência e emergência. J. res.: fundam. care. online. [Internet] 2015;7(3) [acesso em 31 jan 2016]. Disponível: http://dx.doi.org/10.9789/2175-5361.2015.v7i3.2749-2760.

8. Warshawsky NE, Havens DS. Global use of the practice environment scale of the Nursing Work Index. Nurs Res. [Internet] 2011;60(1) [acesso em 23 dez 2015]. Disponível: http://dx.doi.org/10.1097/NNR.0b013e3181ffa79c.

9. Aiken LH, Patrician PA. Measuring organizational traits of hospitals: the revise Nursing work index. Nurs Res. [Internet] 2000;49(3) [acesso em 23 dez 2015]. Disponível: http://journals.Iww.com/nursingresearchonline/pages/ articleviewer.aspx?year $=2000 \& i s s u e=05000 \&$ article $=00006 \&$ type $=$ abstract.

10. Barreto IS, Krempel MC, de Humerez DC. O Cofen e a Enfermagem na América Latina. Enferm Foco. [Internet] 2011;2(4) [acesso em 23 dez 2015]. Disponível: http://revista.portalcofen.gov.br/index.php/enfermagem/article/ viewFile/195/131.

11. Jodas DA, Haddad MCL. Burnout Syndrome among nursing staff from an emergency department of a hniversity hospital. Acta paul. enferm. [Internet] 2009;22(2) [acesso em 23 dez 2015]. Disponível: http://dx.doi. org/10.1590/S0103-21002009000200012.

12. Fontana RT, Brigo L. Estudar e trabalhar: percepções de técnicos de enfermagem sobre esta escolha. Esc Anna Nery. [Internet] 2012;16(1) [acesso em 23 dez 2015]. Disponível: http://dx.doi.org/10.1590/S141481452012000100017.

13. Moceri JT. Hispanic nurses's experiences of bias in the workplace. J Transcult Nurs. [Internet] 2014;25(1) [acesso em 23 dez 2015]. Disponível: http://dx.doi.org/10.1177/1043659613504109.

14. de Jesus MS, Said FA. Autonomia e a prática assistencial do enfermeiro. Cogitare Enferm. [Internet] 2008;13(3) [acesso em 23 dez 2015]. Disponível: http://dx.doi.org/10.5380/ce.v13i3.12996.

15. dos Santos JLG, Erdmann AL, de Andrade SR, de Mello ALSF, de Lima SBS, Pestana AL. Nursing governance: an integrative review of the literature. Rev. esc. enferm. USP. [Internet] 2013;47(6) [acesso em 23 dez 2015]. Disponível: http://dx.doi.org/10.1590/S0080-623420130000600024. 
16. de Oliveira AM, Lemes AM, Machado CR, Loyola e Silva F, Miranda FS. Relação entre enfermeiros e médicos em hospital escola: a perspectiva dos médicos. Rev. Bras. Saúde Mater. Infant. [Internet] 2010;10(2) [acesso em 23 dez 2015]. Disponível: http://dx.doi.org/10.1590/S1519-38292010000600023.

17. Panunto MR, Guirardello EB. Professional nursing practice: environment and emotional exhaustion among intensive care nurses. Rev Latino-Am Enferm. [Internet] 2013;21(3) [acesso em 23 dez 2015]. Disponível: http:// dx.doi.org/10.1590/S0104-11692013000300016.

18. Choi J, Flynn L, Aiken, LH. Nursing practice environment and registered nurses' job satisfaction in nursing homes. Gerontologist. [Internet] 2012;52(4) [acesso em 23 dez 2015]. Disponível: http://dx.doi.org/10.1093/geront/ gnr101. 